nach Bestrahlung (7000 R) und cytostatischer Behandlung mit Dactinomycin, Adriamycin, Vincristin und Cyclophosphamid für 2 Jahre.

Wir führen unsere Behandlung in Anlehnung an das Konzept von HUSTU wie folgt durch:

Bestrahlung mit 5.000 bis 6.000 R Henddosis unter Telekobaltbedingungen auf den erkrankten Knochen und das umgebende Weichteilgewebe. In den ersten 6 Wochen mach Diagnosestellung wöchentlich $500 \mathrm{mg} / \mathrm{m}^{2}$ Cyclophosphamid (Endo- xan) i. $v$, und $1,5 \mathrm{mg} / \mathrm{m}^{2}$ Vincristin i. $v$. Anschließend zwei-wöchentlich 200 bis $500 \mathrm{mg} / \mathrm{m}^{2}$ Cyclophosphamid und 0,75 bis $1,5 \mathrm{mg} / \mathrm{m}^{2}$ Vincristin i. v. Die Dosierung richtet sich außerdem nach den Leukozytenzahlen (10).

Es läBt sich also sagen, daß sich nicht nur nach unseren Erfahrungen EwingSarkome mit konservativer, cytostatischer und radiologischer Therapie besser behandeln lassen als radikal-chirurgisch. Sie sollten daher so gut wie immer konservativ behandelt werden. Alle anderen Knochensarkome spre- chen auf diese Behandlung nicht nachhaltig genug an, die Therapie der Wahl ist hierbei nach wie vor die radikalchirurgische, die durch strahlenthera. peutische Maßnahmen und gelegentlich auch durch Cytostatika komplettiert werden kann. Bei den Knochenmetastasen steht die Rückgewinnung der Statik im Vordergrund. Verbundosteosynthesen und Endoprothesen haben hier in den letzten Jahren bessere Ergebnisse gebracht, als es die radikal-chirurgischen Eingriffe im Rahmen der palliativen Therapie konnten.
Literaturverxeichnis:

1. AAKHUS, T., O. EIDE, K. HOEG und E. POPPE: Das Osteosarkom. Acta chir. scand. $126,520(1963)$

ACCHIAPATTI, G., G. RANDELLI und M. RANDELLI: Betrachiungen über das knochenbildende Sarkom. Arch. Orthop. (Milano) 78, 57 (1965)

3. ADLER, C.-P., S. WELLER und A. KLOMPER: Maligne Knochentumoren und Weichteilsarkome des Bewegungsapparates. Bruns' Beitr. klin. Chir. 217, 1 (1969)

4. CORTES, E P. J. F. HOLLANO, J. J. WANG L. F. SINKS, J. BLOM, H. SENN, A. BANK, and $O$. GLIDEWELL: Amputation and Adriamycin in Primary Osteosarcoma. New Engl. 1. Med. 291, $998-7000(1974)$.

5. FALK, S., und M. ALPERT: Klinische und rontgenologische Aspekte des Ewing-Sarkoms. Amer. J. med. Sci. 250, 492 (1965)

6. FLEISSNER, H. K.: Analyse der in der Orthooädischen klinik der KarlMarx-Universität Leipzig von 1956 bns 1967 beobachteten Knodientumoren. Beitr. Orthop. 16, 787 (1969)

7. GOIDANICH, I. F.: Beobachtungen von 100 Osteosarkomen, die im orthopädischen Irstitut Rizzoli in Bologna behandelt wurden. Acta chir. orthop. padischen .

8. GOLOSHTEIN mit Knochensarkomen in Leningrad. Zbl. Chir. 2291 (1980)

9. HAAS, F., R. IUNGBLUT und F. HEINZLER: Die Strahlenbehandlung des Ewing-Sarkoms Strahlentherapis 180 133 (1970)

HUSTU H. O. D. PINKEL, and C. B. PRATT: Treatment of Cinical tocalized Euring's Sarcoma with Radiotherapy and Combination Chemo-

therapy. Cancer $30,1522-1527$ (1972).

schwilste und Weichteilsarkome. Strahlentherapie 137, 253 (1969)
12. JAFFE, $N$, E FREI, III, D. TRAGGIS, and $Y$, BISHOP: Adiuvant Methotrexate and Citrovorum. Factor Freatment of Osteogenic Sarcoma. New Eng. J. Med. 291, $994-997$ (1974)

13. LAMPERT, F.: Krebs im Kindesalter. 3. Aufl. Urban und Schwarzenberg München - Berlin - Wien (1974)

14. MALPAS, J. S. and 3. E. FREEMAN: Solid Tumors in Children. Brit. Med. $7.710-713(1974)$

15. MCKENNA, R, J, C. P. SCHWINN, K. Y. SOONG und N. C. HIGIN BOTHAM: Sarkome der osteogenetischen Reihe. Eine Analyse von 552 Fällen. 3 . Bone It Surg. $48-4,1$ (1966)

16. NESBIT, M. E.: Bone Tumors in Infants and Children. Paediatrician 1, $273-287(1972 / 73)$

17. NEUBERT, $C$., und $H$. ECKE: Nachuntersuchungsergebnisse und Prognose unterschiedich behandelter sarkomatöser Geschwülste der Extremitäten sounterchicdich

8. OTT, G., und R. FREY: Klinische Beobachtungen und statistische Auswertung bei 780 Sarkomkranken. Arch. klin. Chir. 295, 971 (1960)

19. OT, $G$ und $R$ FREY: Klinik, Behandlung und Statistik der Sarkome Erg. Chir. Orth. 43, $410(1961)$

20. ROSEN, G. N. WOLLNER, C TAN, S. J HAJDU W CHAM G J DANGIO and M MURPHY: Disease-free Survival in Children with Ewing's Sarcome Treated with Radiation Therapy and Adjuvant four-drug Sequential Chamo theraoy Cancer $33,384-393(1974)$

21. SWEETNAM R. Tumours of Bone and their Management Ann. roy. Coll. Surg. Engl. $5 \phi_{,} 63$ (1974)

22. WEHNER, W: Das Vorgehen bei Skelettmetastasen. Beitr. Orthop. 17, (1970).

Anschrift der Verlasser:

Für das Zentrum Chirurgie: Or. Chr. NEUBERT

Fur das Zentrum Chirurgie: Or. Chr. NEUBERT

Justus-Liebig-Universität Gießen

\title{
Buchbesprechung
}

\section{Operationen im Kindesalter}

Ein Atlas in zwei Bänden

Herausgegeben von Prof. Dr. H. KUNZ, Wien

Band II: Kopf und Hals, Neurochirurgie, Orthopädische Eingriffe, Mißbildungen im Handbereich, Osteomyelibis, Urologie. Nebennieren

Mit Beiträgen von K. Chiani, G. Freilinger, R. Hohenfellner, W. Hoppe, L Jani, H. Kraus, E. Morscher, B. Spiessl, K Srockamo E Siraub M. SunderPlaßmann, D. Waigand, H.D. Wulff 1975. XII, 396 Seiten, 455 Abbildungen in 597 Einzeldarstelfungen

(Oeorg Thieme Verlag, Suttgart.)

Format $20 \times 28 \mathrm{~cm}$

Ganzl. DM 248-.

(ISBN 313479701 1)

Ausgeliefert am: 15. Mai 1975
Unter der Herausgeberschaft von $H$. KUNZ haben namhafte Autoren den vorliegenden 2. Band des Atlasses der Operationen im Kindesalter konzipiert.

Das Buch gliedert sich in Operationen am Kopf und Hals, im Rahmen der Neurochirurgie, der Orthopädie, der MiBbildungen im Handbereich, der Osteomyelitis, der Urologie und die Operationen an den Nebennieren. Die einzelnen Kapitel sind bezüglich ihrer Indikation, der Operationsmethoden und ihrer Technik klar gegliedert und mit zahtreichen instruktiven Situationszeichnungen, Strichzeichnungen und Fotografien ausgestatteł. Besonders begrüBenswert sind plastisch-chirurgische Eingriffe im Bereich des kindlichen Kopfes und die unterschiedlichen Methodiken der Arthroplastik am Hüftgelenk dargestellt. Auch die in der Praxis manchmal recht schwierig $z u$ beseitigenden angeborenen Fehlbildungen im Handbereich und der möglichen operativen Hilfe sprechen in den Wark sehr an.

Der zweite Band engänzt und rundet das Stoffgebiet des ersten ab. Beide Bände zusammen, die vom gleichen Herausgeber, aber unterschiedlichen Autoren konzipiert worden sind, wirken wie eus einem Stück gegossen und geben gemeinsam einen Oberblick über die notwendigen Eingriffe am Kird. Sie sind auch im Detall der Vopbereitung der Operationsolanung und technik sowie der Schilderung der Fehtermöglichkeiten, die sich einschleichen können, aus einem GuB. Beide Atlanten gehören in die Hände der operativ tätigen und interessierten Ärzte, gleichgültig ob sie noch in der Ausbildung stehen oder bereits ihre jeweiligen Fachgebiete übersehen. Für die Studierenden empfehlen sich beide Atlanten als Nachschlagewerk, sie sollten deshalb auch in keiner Institutsbibliothek fehlen. 